\title{
THE HIGH-TEMPERATURE BEHAVIOUR OF LATTICE QCD WITH FERMIONS
}

\author{
J. ENGELS, F. KARSCH and H. SATZ \\ Fakultät für Physik, Universität Bielefeld, Bielefeld, Germany
}

Received 29 March 1982

\begin{abstract}
By Monte Carlo simulation on the lattice, we calculate the high-temperature behaviour of the energy density $\epsilon$ in SU(2) and SU(3) QCD with Wilson fermions. From the leading term of the hopping parameter expansion, we find that $\epsilon$ converges very rapidly to the Stefan-Boltzmann limit of an asymptotically free quark-gluon gas. The behaviour in the non-asymptotic region indicates that chiral symmetry restoration occurs at a higher temperature $\left(T_{\mathrm{ch}}\right)$ than colour deconfinement $\left(T_{\mathrm{c}}\right)$, with $T_{\mathrm{ch}} / T_{\mathrm{c}} \approx 1.3$
\end{abstract}

With increasing temperature, strongly interacting matter is expected to undergo a phase transition from a state of confined quarks and gluons to a state in which colour screening renders the constituents effectively free.

For pure Yang-Mills systems, it was shown by Monte Carlo simulation on the lattice that this asymptotically free behaviour is in fact attained rather quickly $[1-4]$. The deconfinement transition was found to occur at $[1-8]$

$$
\begin{aligned}
T_{\mathrm{c}} & =38-43 \Lambda_{\mathrm{L}} \approx 0.41-0.47 \sigma^{1 / 2}, \quad \operatorname{SU}(2), \\
& =75-83 \Lambda_{\mathrm{L}} \approx 0.38-0.42 \sigma^{1 / 2}, \quad \operatorname{SU}(3),
\end{aligned}
$$

for colour SU(2) and SU(3), respectively. Here $\Lambda_{\mathbf{L}}$ denotes in each case the lattice scale, which is changed into physical units by relating it to the string tension $[9,10] \sigma=0.16 \mathrm{GeV}^{2}$. At temperatures of less than $2 T_{\mathrm{c}}$, the energy density $\epsilon$ for both $\mathrm{SU}(2)$ and $\mathrm{SU}(3)$ systems has already approached the asymptotic Stefan-Boltzmann limit,

$$
\begin{array}{rlrl}
\epsilon_{\mathrm{SB}} / T^{4} & =\pi^{2} / 5, & & \mathrm{SU}(2), \\
& =8 \pi^{2} / 15, & \mathrm{SU}(3),
\end{array}
$$

to within $10-15 \%$.

In the present note we want to give first results obtained in extending these considerations to QCD with fermions, both for $\mathrm{SU}(2)$ and SU(3) colour groups. The Stefan-Boltzmann form in these cases becomes

$$
\begin{array}{rlrl}
\epsilon_{\mathrm{SB}} / T^{4} & =\pi^{2} / 5+7 \pi^{2} / 15, & & \mathrm{SU}(2), \\
& =8 \pi^{2} / 15+7 \pi^{2} / 10, & \mathrm{SU}(3),
\end{array}
$$

where the first term corresponds to the gluon and the second to the quark-antiquark component, with two quark flavours. We want to investigate in the following to what extent high-temperature Monte Carlo lattice calculations approach these values.

The QCD lagrangian density for massless quarks of one flavour only can be written

$$
\begin{aligned}
& \mathcal{L}(\psi, A)=-\frac{1}{4} F_{\mu \nu}^{a} F_{a}^{\mu \nu}+\bar{\psi}_{\alpha}\left(\mathrm{i} \not{\phi}-g A_{a} \lambda^{a}\right)_{\alpha \beta} \psi_{\beta}, \\
& F_{\mu \nu}^{a}=\partial_{\mu} A_{\nu}^{a}-\partial_{\nu} A_{\mu}^{a}-g f_{b c}^{a} A_{\mu}^{b} A_{\nu}^{c} .
\end{aligned}
$$

Here $\mu, \nu$ denote tensor and $\alpha, \beta$ spinor indices; $a, b, c$ are colour indices, and $f_{b c}^{a}$ is the structure function of the $\mathrm{SU}(N)$ gauge group. The finite-temperature euclidean action becomes

$S_{\beta}(\psi, A)=-\int \mathrm{d}^{3} x \int_{0}^{\beta} \mathrm{d} \tau \mathcal{L}(\psi, A)$,

with periodic (antiperiodic) boundary conditions in the temperature integration of the boson (fermion) fields. The action is a sum

$S_{\beta}(\psi, A)=S_{\beta}^{\mathrm{G}}(A)+S_{\beta}^{\mathrm{F}}(\psi, A)$,

of the pure Yang-Mills part $S_{\beta}^{\mathrm{G}}$ and the quark-gluon part $S_{\beta}^{\mathrm{F}}$. Since the thermodynamics of the $\mathrm{SU}(N)$ 
Yang-Mills system is treated in detail elsewhere [2], we shall concentrate here on $S_{\beta}^{\mathrm{F}}$.

Fermion theories on the lattice lead to species doubling [11], unless one is willing to accept chiral symmetry breaking [12], non-local derivatives [13] or perhaps random lattice structure [14]. We shall here use Wilson's form [12], in which chiral symmetry is recovered only in the continuum limit. We consider an asymmetric lattice, with $N_{\sigma}$ spatial and $N_{\beta}$ temporal sites. Temperature $T=\beta^{-1}$ and spatial volume $V$ are defined as

$\beta=N_{\beta} a_{\beta}, \quad V=\left(N_{\sigma} a_{\sigma}\right)^{3}$,

with $a_{\beta}\left(a_{\sigma}\right)$ denoting the temporal (spatial) lattice spacing; in actual calculations, we shall generally choose $\xi \equiv a_{\sigma} / a_{\beta}=1$.

On this lattice, the action $S^{\mathrm{F}}$ of the quark-gluon sector is written [12]

$$
\begin{aligned}
S^{\mathrm{F}} & =\sum_{n}\left(\bar{\psi}_{n} \psi_{n}-K_{\beta}\left[\bar{\psi}_{n}\left(1-\gamma_{0}\right) U_{n, n+\hat{0}} \psi_{n+\hat{0}}\right.\right. \\
& \left.+\bar{\psi}_{n-\hat{0}}\left(1+\gamma_{0}\right) U_{n-\hat{0}, n}^{+} \psi_{n}\right] \\
& -K_{\sigma} \sum_{\mu=1}^{3}\left[\bar{\psi}_{n}\left(1-\gamma_{\mu}\right) U_{n, n+\hat{\mu}} \psi_{n+\hat{\mu}}\right. \\
& \left.\left.+\bar{\psi}_{n-\hat{\mu}}\left(1+\gamma_{\mu}\right) U_{n-\hat{\mu}, n}^{+} \psi_{n}\right]\right),
\end{aligned}
$$

where we have suppressed all but symbolic lattice indices $n, m$. The $K_{\beta}$ term refers to that part of the lattice summation in which the gauge group elements $U_{n m}$ are associated with timelike lattice links $n, m$; for $K_{\sigma}$, the links are spacelike. On an isotropic lattice, $K_{\beta}\left(g^{2}, \xi=1\right)=K_{\sigma}\left(g^{2}, \xi=1\right)=K\left(g^{2}\right)$ is the usual "hopping" parameter $[12,15]$.

For the pure $\mathrm{SU}(N)$ Yang-Mills part, we have [2]

$$
\begin{aligned}
S^{\mathrm{G}} & =2 N\left(K_{\beta}^{\mathrm{G}} \sum_{\left\{P_{\beta}\right\}}\left(1-N^{-1} \operatorname{Re} \operatorname{tr} U_{i j} U_{j k} U_{k l} U_{l i}\right)\right. \\
& \left.+K_{\sigma}^{\mathrm{G}} \sum_{\left\{P_{\sigma}\right\}}\left(1-N^{-1} \operatorname{Re} \operatorname{tr} U_{i j} U_{j k} U_{k l} U_{l i}\right)\right),
\end{aligned}
$$

In terms of $S^{\mathrm{F}}$ and $S^{\mathrm{G}}$, the euclidean form of the QCD partition function on the lattice is now given by

$$
\begin{aligned}
Z_{\mathrm{E}} & =\int \prod_{\mathbf{L}} \mathrm{d} U \prod_{\mathbf{S}} \mathrm{d} \psi \mathrm{d} \bar{\psi} \\
& \times \exp \left[-S^{\mathrm{G}}(U)-S^{\mathrm{F}}(U, \psi, \bar{\psi})\right],
\end{aligned}
$$

with the $\mathrm{d} U$ integration to be carried out for all links, the $d \psi d \bar{\psi}$ integrations for all sites of the lattice.

Since the fermion action $S^{\mathrm{F}}$ has the form

$$
\begin{aligned}
& S^{\mathrm{F}}=\bar{\psi}(1-K M) \psi, \\
& M_{\mu}(U)=\left(1-\gamma_{\mu}\right) U_{n m} \delta_{n, m-\hat{\mu}} \\
& \quad+\left(1+\gamma_{\mu}\right) U_{m n}^{+} \delta_{n, m+\hat{\mu}},
\end{aligned}
$$

the integration over the anti-commuting spinor fields can be carried out [16] to give an effective boson form

$Z_{\mathrm{E}}=\int \prod_{\mathrm{L}} \mathrm{d} U \exp \left[-S^{\mathrm{G}}(U)\right] \operatorname{det}(1-K M)$,

note that in our case $K$ is "direction dependent":

$K M=K_{\beta} M_{0}+K_{\sigma} \sum_{\mu=1}^{3} M_{\mu}$.

Note further that because of the antiperiodic boundary condition required for the fermion fields, in eq. (14) $\delta_{n, m \pm \hat{0}}$ has to be replaced by $-\delta_{n, m \pm 0}$ whenever the lattice points are joined by a link which touches the end of the lattice in the temperature direction.

The euclidean energy density $\epsilon_{\mathrm{E}}$, which after subtraction of the vacuum contribution gives the physical energy density $\epsilon$, is obtained from $Z_{\mathrm{E}}$ by

$$
\begin{aligned}
\epsilon_{\mathrm{E}} & =-V^{-1}\left(\partial \ln Z_{\mathrm{E}} / \partial \beta\right)_{V} \\
& =\left[\xi^{2} /\left(N_{\sigma}^{3} N_{\beta} a_{\sigma}^{4}\right)\right]\left(\partial \ln Z_{\mathrm{E}} / \partial \xi\right)_{a_{\sigma}} .
\end{aligned}
$$

It also becomes the sum

$\epsilon_{\mathrm{E}}=\epsilon \mathrm{G}+\epsilon_{\mathrm{E}}^{\mathrm{F}}$

of a pure gluon part where $\left\{P_{\beta}\right\}\left(\left\{P_{o}\right\}\right)$ denotes the summation over space-time (space-space) plaquettes, and $K_{\beta}^{\mathrm{G}}, K_{\sigma}^{\mathrm{G}}$ the corresponding gluon couplings. 


$$
\begin{aligned}
\epsilon_{\mathrm{E}}^{\mathrm{G}} & =-\left[2 N \xi^{2} /\left(N_{\sigma}^{3} N_{\beta} a_{\sigma}^{4} Z_{\mathrm{E}}\right)\right] \\
& \times \int \prod_{\mathrm{L}} \mathrm{d} U \exp \left[-S^{\mathrm{G}}(U)\right] \operatorname{det} Q \\
& \times\left(\left(\partial K_{\beta}^{\mathrm{G}} / \partial \xi\right)_{a_{\sigma}} \sum_{\left\{P_{\beta}\right\}}\left(1-N^{-1} \operatorname{Re} \operatorname{tr} U U U U\right)\right. \\
& \left.+\left(\partial K_{\sigma}^{\mathrm{G}} / \partial \xi\right)_{a_{\sigma}} \sum_{\left\{P_{\sigma}\right\}}\left(1-N^{-1} \operatorname{Re} \operatorname{tr} U U U U\right)\right)
\end{aligned}
$$

and a quark-gluon part

$$
\begin{aligned}
\epsilon_{\mathrm{E}}^{\mathrm{F}} & =-\left[\xi^{2} /\left(N_{\sigma}^{3} N_{\beta} a_{\sigma}^{4} Z_{\mathrm{E}}\right)\right] \\
& \times \int \prod_{\mathrm{L}} \mathrm{d} U \exp \left[-S^{\mathrm{G}}(U)\right] \operatorname{det} Q \\
& \times\left(\left(\partial K_{\beta} / \partial \xi\right)_{a_{\sigma}} \operatorname{tr}\left(M_{0} Q^{-1}\right)\right. \\
& \left.+\left(\partial K_{\sigma} / \partial \xi\right)_{a_{\sigma}} \sum_{\mu=1}^{3} \operatorname{tr}\left(M_{\mu} Q^{-1}\right)\right),
\end{aligned}
$$

with $Q \equiv 1-K M(U)$.

The computational problem beyond what is encountered in the pure Yang-Mills case lies in the evaluation of det $Q$ and of $Q^{-1}$. We shall here use the expansion of these quantities in powers of the fermionic couplings $K_{\beta}$ and $K_{\sigma}$ ("hopping parameter expansion" [15]), and retain in both cases only the leading term. By calculating an ideal gas of massless fermions in the same approximation, we shall then get some idea of how valid this procedure may be.

For $\operatorname{det} Q$ the leading term is

$\operatorname{det} Q=\operatorname{det}(1-K M) \approx 1$,

("quenched approximation" [17]), while in the expansion

$Q^{-1}=[1-K M(U)]^{-1}=\sum_{l=0}^{\infty} K^{l} M(U)^{l}$,

because of gauge invariance the first contribution to $\operatorname{tr}\left(Q^{-1} M\right)$ arises for the shortest non-vanishing closed loop obtained from $M(U) \sim U$. For $N_{\beta}=2$ and 3, this is a thermal loop, i.e. one closed in the temperature direction; hence in that case, the first term is $l=$ $N_{\beta}-1$. For $N_{\beta} \geqslant 4$, these loops are not the only ones; but the non-thermal loops after subtraction of the vacuum term lead to negligibly small contributions, so that we obtain on an isotropic lattice $(\xi=1)$

$\epsilon^{\mathrm{F}} a^{4} \approx\left(\partial K_{\beta} / \partial \xi\right)_{\xi=1} K^{N_{\beta}-1} 2^{N_{\beta}+2}\langle L\rangle$,

with $\langle L\rangle$ for the expectation value of the thermal Wilson loop, and $a$ for the lattice spacing. In the quenched approximation there is still a global $Z_{N}$ symmetry of the action, which is spontaneously broken at high temperatures [6-8]. Therefore $N$ equivalent, but different link configurations of the system are possible. Only one of them is connected to the physical continuum limit, where $U \rightarrow 1$. The corresponding $\langle L\rangle$ is real and positive. In the case of a non-interacting $\mathrm{SU}(N)$ system, we have $\langle L\rangle=N$.

To test the convergence of the hopping parameter expansion, we compare in table 1 the value of the leading term for an ideal gas of massless quarks with the exact result [18] for such a system, both calculated on lattices of the same size. We see that for low $N_{\beta}$ values, the approximation given by just the leading term is quite reasonable. This leads us to hope that also for SU(2) and SU(3) QCD we can obtain an indicative estimate by retaining that term only.

We now return to eq. (22) for the quark-gluon energy density of SU( $N)$ QCD. The fermion coupling $K\left(g^{2}\right)$ for massless quarks on a symmetric, isotropic lattice $\left(N_{\sigma}=N_{\beta}, \xi=1\right)$ has been evaluated numerically both at large [19] and at small [20] $g^{2}$. The thermal Wilson loop $\langle L\rangle$ can be calculated by the usual finite-temperature Monte Carlo technique [1-8]; for SU(2) we have carried out this calculation on different size lattices, while for $\mathrm{SU}(3)$ we shall use the results from ref. [3]. To evaluate eq. (22), we then still need $\left(\partial K_{\beta} / \partial \xi\right)_{\xi=1}$.

We now write

$K_{\beta}=\frac{1}{2}[\xi /(3+\xi)] k\left(\xi, g^{2}\right)$,

Table 1

Ratio $R$ of the leading term of the hopping parameter expansion for the energy density of an ideal gas of massless fermions to the exact energy density on an infinite spatial lattice and several $N_{\beta}$ values.

\begin{tabular}{ll}
\hline$N_{\beta}$ & $R$ \\
\hline 2 & 1.086 \\
3 & 0.944 \\
4 & 0.764 \\
5 & 0.557 \\
\hline
\end{tabular}


where $k\left(\xi, g^{2}\right)$ is unity for a gas of non-interacting massless fermions [18]. As in the case of the pure Yang-Mills system [21], we here expect $k\left(\xi, g^{2}\right)$ to be only very weakly dependent on $\xi$, if $g^{2}$ is sufficiently small; for $g^{2} \rightarrow 0$, one has [20]

$k\left(\xi, g^{2}\right)=1+f(\xi) g^{2}$.

The additional weak $\xi$ dependence arising from $k(\xi, g)$ can in principle be determined as in ref. [21]; we shall here neglect it to get

$\left(\partial K_{\beta} / \partial \xi\right)_{\xi=1} \approx \frac{3}{32} k\left(1, g^{2}\right)=\frac{3}{4} K\left(g^{2}\right)$.

With the connection between $g^{2}$, the lattice spacing $a$ and the lattice scale $\Lambda_{\mathrm{L}}$, as given by the renormalization group relation ${ }^{\neq 1}$

$a \Lambda_{\mathrm{L}}=\exp \left[-24 \pi^{2} / 11 N g^{2}-\frac{51}{121} \ln \left(11 N g^{2} / 48 \pi^{2}\right)\right]$,

we can then obtain $\epsilon^{\mathrm{F}}$ as function of the temperature $T=\beta^{-1}=\left(N_{\beta} a\right)^{-1}$.

Let us now consider this procedure in more detail. Comparing the leading term of the hopping parameter expansion for $\epsilon^{\mathrm{F}}$ with that of an ideal gas of massless fermions, $\epsilon \mathrm{SB}$, we obtain from eqs. (22) and (25)

$\epsilon^{\mathrm{F}} / \epsilon_{\mathrm{SB}}^{\mathrm{F}}=\left[8 K\left(g^{2}\right)\right]^{N_{\beta\langle L}} / N$,

where we have used $K=1 / 8,\langle L\rangle=N$ for the ideal gas analog of the $\mathrm{SU}(N)$ case.

The fermion coupling $K\left(g^{2}\right)$ is for small $g^{2}$ approximated by [20]

$K\left(g^{2}\right)=\frac{1}{8}\left\{1+0.074\left[\left(N^{2}-1\right) / 2 N\right] g^{2}\right\}$

for SU(3), there are Monte Carlo results for large $g^{2}$ down to $g^{2} \approx 1$ [19], suggesting that eq. (28) gives for massless quarks a lower bound for $K\left(g^{2}\right)$ in that range of $g^{2}$.

We now first tabulate in table 2 the results for the $\mathrm{SU}(2)$ case, with eq. (28) for $K\left(\mathrm{~g}^{2}\right)$, and our data for $\langle L\rangle$. We note that above $T \sim 90 \Lambda_{\mathrm{L}}$, the system behaves essentially like an ideal gas, although neither $K$ nor $\langle L\rangle$ have attained their ideal gas values. We further note that this is the case for different $N_{\beta}$ as well - the change in $\langle L\rangle$ is just compensated sufficiently by the coupling factor to retain asymptotically free behaviour. A continuation to lower values

\#1 Eq. (26) should only be used in the quenched approximation. For a discussion of this point see ref. [17].
Table 2

Hopping parameter $K$, thermal Wilson loop $\langle L\rangle$ and ratio ${ }_{\epsilon} \mathrm{F} / \epsilon_{\mathrm{SB}}^{\mathrm{F}}$ calculated from eq. (27) as function of the temperature [eq. (26)] for $\mathrm{SU}(2)$ and various $N_{\beta}$.

\begin{tabular}{lrlll}
\hline$N_{\beta}$ & $T / \Lambda_{\mathrm{L}}$ & $8 K$ & $\langle L\rangle$ & $\epsilon / \epsilon$ SB \\
\hline 2 & 60 & 1.103 & 1.113 & 0.667 \\
& 100 & 1.094 & 1.251 & 0.748 \\
& 300 & 1.079 & 1.419 & 0.826 \\
& 500 & 1.074 & 1.465 & 0.845 \\
& 1000 & 1.068 & 1.519 & 0.866 \\
3 & 93 & 1.089 & 0.987 & 0.637 \\
& 330 & 1.074 & 1.182 & 0.732 \\
& 1200 & 1.063 & 1.276 & 0.767 \\
& 4330 & 1.056 & 1.405 & 0.826 \\
4 & 60 & 1.091 & 0.595 & 0.422 \\
& 100 & 1.084 & 0.775 & 0.535 \\
& 300 & 1.072 & 0.987 & 0.652 \\
& 500 & 1.068 & 1.058 & 0.688 \\
& 1000 & 1.063 & 1.134 & 0.723 \\
\hline
\end{tabular}

of $T$ requires a Monte Carlo evaluation of $K\left(\mathrm{~g}^{2}\right)$ for the SU(2) case, since eq. (28) then breaks down, because on a lattice with small $N_{\beta}$ we need larger values of $g^{2}$ for a given temperature. In fig. 1 , we show the temperature dependence of $\epsilon^{\mathrm{F}} / \epsilon_{\mathrm{SB}}^{\mathrm{F}}$, combining the results from the different lattice sizes. If the temperature is decreased below $90 \Lambda_{\mathrm{L}}$, there is a definite fall-off towards confinement; however, for a meaningful continuation on lattices with $N_{\beta} \leqslant 4$, we need a better form of $K\left(g^{2}\right)$ than eq. (28). Such an improved form could be obtained either numerically, as in ref. [19], or perhaps by inclusion of higher orders of $g^{2}$ in eq. (28).

We now come to the SU(3) case, which is obviously the physically most interesting one. In table 3 , we display, for $N_{\beta}=3$ and 4 , the values ${ }^{\ddagger 2}$ of $\langle L\rangle$ from ref. [3], together with the coupling $K\left(g^{2}\right)$, which is taken from the $u, d$ form of ref. [19], and the resulting energy density ratio $\epsilon^{\mathrm{F}} / \epsilon_{\mathrm{SB}}^{\mathrm{F}}$. We note that here also the energy density very quickly approaches its asymptotic value - and again not because $K$ and $\langle L\rangle$ separately do so, but rather because these densities, for each $N_{\beta}$, together provide an almost asymptotic energy density. In fig. 2 we display the temperature behaviour of the combined $N_{\beta}=3$ and 4 results. Here

$\$ 2$ The values of $\langle L\rangle$ were obtained from the $\langle L\rangle^{3}$ values of ref. [3] by taking the real third root of these values. 


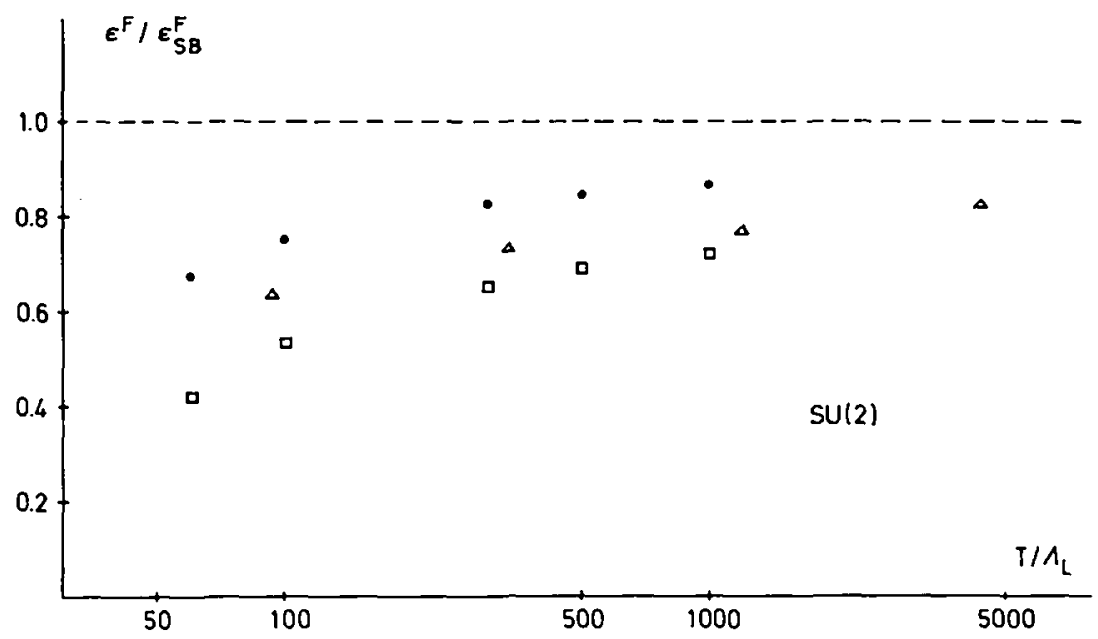

Fig. 1. High-temperature behaviour of the ratio $c \% / e \mathrm{SB}$ according to eqs. (27) and (28) for $\mathrm{SU}(2)$ and $N_{\beta}=2$ (full points), 3 (triangles) and 4 (squares).

we can, because of the large $g^{2}$ values of the fermion coupling obtained in ref. [19], go to somewhat lower temperatures than in the SU(2) case. We note a sharp drop around $T \sim 80 \Lambda_{\mathrm{L}}\left(\sim 0.4 \sigma^{1 / 2}\right)$, which presumably corresponds to the onset of confinement.

In fig. 3, we show the overall energy density $\epsilon / T^{4}$, obtained by combining our above results for $\epsilon^{F}$ with the pure Yang-Mills results of ref. [4]. We conclude that full quantum chromodynamics with fermions

Table 3

The same quantities as in table 2 for SU(3). The errors in $8 K$ are due to the uncertainties in the determination of $K\left(\mathrm{~g}^{2}\right)$ for $1 / g^{2}>0.95$ in ref. [19].

\begin{tabular}{rrlll}
\hline$N_{\beta}$ & $T / \Lambda_{\mathrm{L}}$ & $8 K$ & $\langle L\rangle$ & $\epsilon^{\mathrm{F}} / \epsilon_{\mathrm{SB}}^{\mathrm{F}}$ \\
\hline 3 & 80 & 1.536 & 0.31 & 0.374 \\
& 84 & 1.512 & 0.63 & 0.726 \\
& 89 & 1.496 & 0.73 & 0.815 \\
& 95 & 1.472 & 0.88 & 0.935 \\
& 100 & 1.456 & 0.96 & 0.988 \\
& 110 & $1.416-1.448$ & 1.04 & $1.01 \pm 0.03$ \\
& 120 & $1.384-1.440$ & 1.08 & $1.02 \pm 0.07$ \\
& 130 & $1.360-1.440$ & 1.13 & $1.04 \pm 0.10$ \\
& 140 & $1.328-1.432$ & 1.17 & $1.03 \pm 0.12$ \\
4 & 76 & 1.456 & 0.29 & 0.434 \\
& 84 & $1.416-1.448$ & 0.60 & $0.84 \pm 0.04$ \\
& 90 & $1.384-1.440$ & 0.64 & $0.85 \pm 0.07$ \\
& 100 & $1.328-1.432$ & 0.73 & $0.89 \pm 0.13$ \\
\hline
\end{tabular}

indeed appears to lead to the deconfinement behaviour observed in the study of Yang-Mills systems alone [1-4].

Finally, we want to look at a specific fermion aspect of the problem, chiral symmetry restoration. We have here used a formulation in which the fermions on the lattice explicitly break chiral invariance, even in the case of a non-interacting system. The appropriate measure for chiral symmetry restoration in such a scheme should therefore be [22] $\langle\bar{\psi} \psi\rangle_{\mathrm{SB}}-$ $\langle\bar{\psi} \psi\rangle$ which vanishes if $\langle\bar{\psi} \psi\rangle$ becomes equal to the result of an ideal gas of massless fermions. Using as above the leading term of the hopping parameter expansion, we obtain for the SU(3) system

$$
\begin{aligned}
{\left[\langle\bar{\psi} \psi\rangle_{\mathrm{SB}}-\langle\bar{\psi} \psi\rangle\right] /\langle\bar{\psi} \psi\rangle_{\mathrm{SB}} } & =1-(8 K)^{N_{\beta}\langle L\rangle / 3,} \\
& =1-\epsilon^{\mathrm{F}} / \epsilon_{\mathrm{SB}}^{\mathrm{F}},
\end{aligned}
$$

if $N_{\beta} \leqslant 3$. The corresponding temperature behaviour (for $N_{\beta}=3$ ), using eq. (26), is shown in fig. 4 . From this we conclude that chiral symmetry is restored for

$T_{\text {ch }} \sim 100 \Lambda_{\mathrm{L}} \simeq 1.3 T_{\mathrm{c}}$,

i.e. at a slightly higher critical temperature than that for colour deconfinement. Such a behaviour, $T_{\mathrm{ch}}>T_{\mathrm{c}}$, would necessarily arise here if colour deconfinement is a continuous phase transition. It has also been proposed in the context of more phenomenological considerations [23]. 


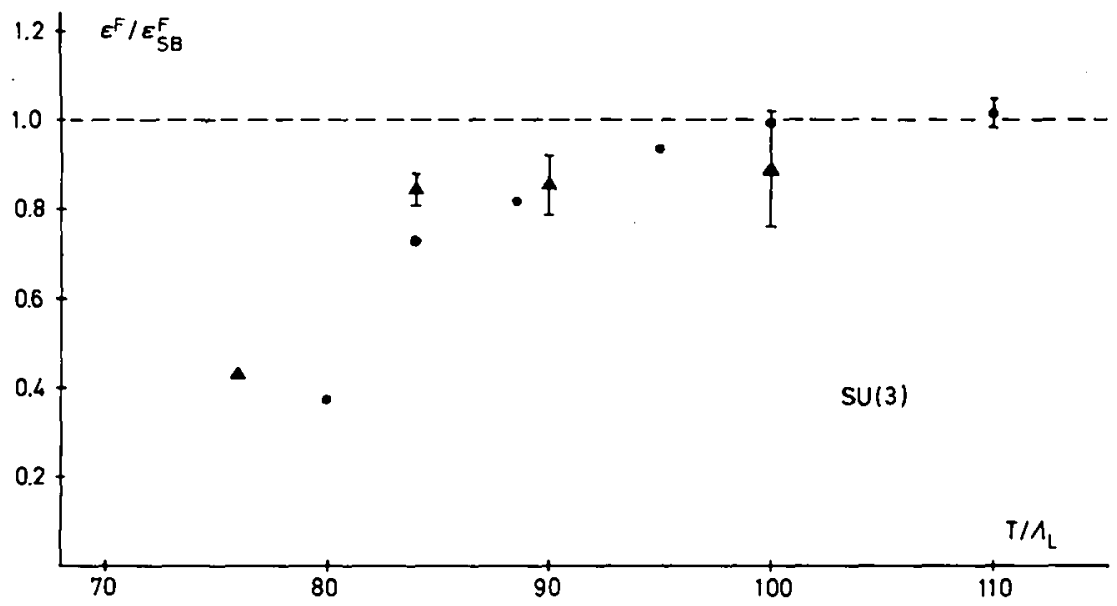

Fig. 2. The ratio $\epsilon / \epsilon \underset{S B}{F}$ from eq. (27) versus temperature for $S U(3)$ and $N_{\beta}=3$ (full points) and 4 (triangles). The errors on the points are those of table 3 .

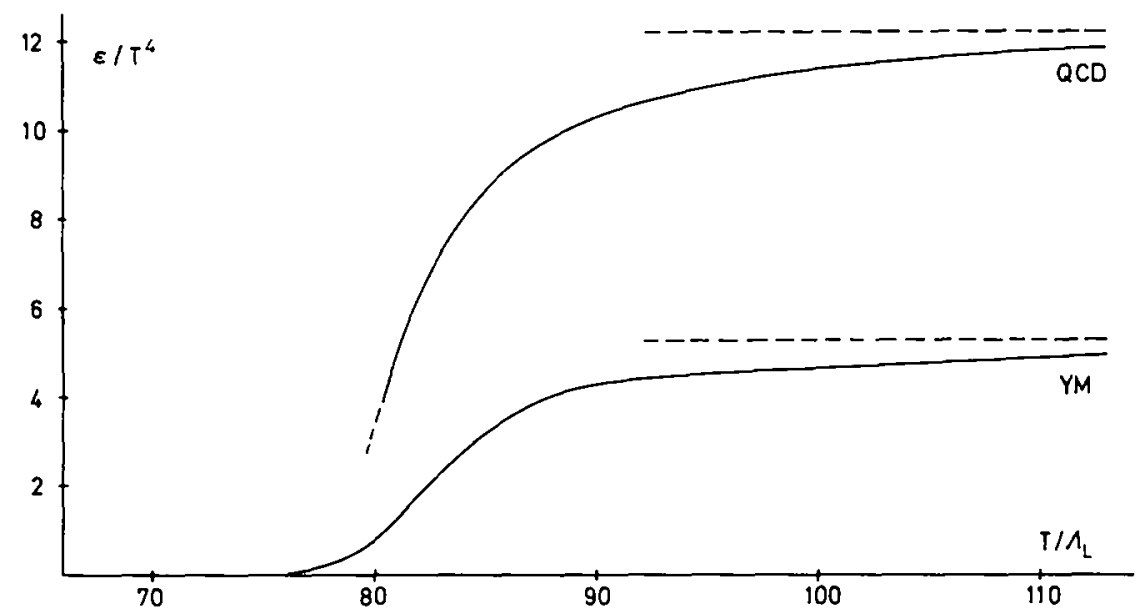

Fig. 3. The full energy density $\epsilon$ divided by $T^{4}$ for $S U(3),(Q C D)$, obtained by combining our results for $\epsilon^{F}$ and those for the pure Yang-Mills system (YM) of ref. [4]. The dashed lines show the corresponding Stefan-Boltzmann limits [eqs. (2) and (3)].

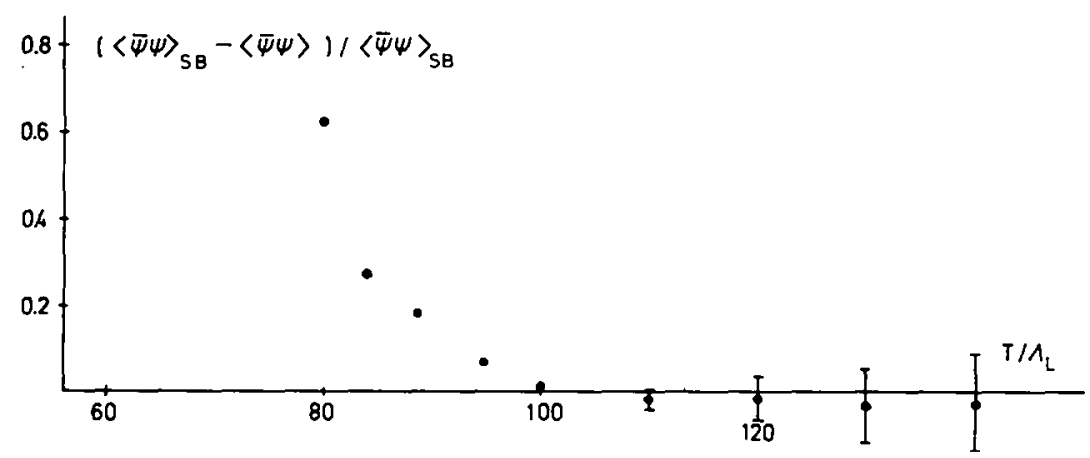

Fig. 4. The ratio $\left.\left[\langle\bar{\psi} \psi\rangle_{\mathrm{SB}}-\langle\bar{\psi} \psi\rangle\right] /\langle\bar{\psi} \psi\rangle\right\rangle_{\mathrm{SB}}$ versus temperature for $\mathrm{SU}(3)$ and $N_{\beta}=3$. 
It is striking that already the lowest order of the hopping parameter expansion leads to such reasonable results. It certainly has to be studied to what extent higher-order terms will induce modifications - in particular, whether the critical temperature $T_{\mathrm{c}}$ for colour deconfinement will remain unchanged after the introduction of virtual quark loops. Our conclusions about chiral symmetry restoration were obtained from a formulation with Wilson fermions; they should be verified by using instead a formulation which already on the lattice has chiral invariance.

Altogether the results obtained here show that the thermodynamic behaviour of QCD systems is obtainable by lattice methods, and our results provide a first picture of this behaviour.

\section{References}

[1] J. Engels, F. Karsch, I. Montvay and H. Satz, Phys. Lett. 101 B (1981) 89.

[2] J. Engels, F. Karsch, I. Montvay and H. Satz, Gauge field thermodynamics for the SU(2) Yang--Mills systcm, Bielefeld preprint, BI-TP 81/29 (December 1981).

[3] I. Montvay and E. Pietarinen, Phys. Lett. 110B (1982) 148.

[4] I. Montvay and E. Pictarinen, Thermodynamical properties of the gluon matter, Helsinki preprint, HU-TFT$82-8$ (1982).

[5] L. McLerran and B. Svetitsky, Phys. Lett. 98B (1981) 195.

[6] L. McLerran and B. Svetitsky, Phys. Rev. D24 (1981) 450.

[7] J. Kuti, J. Polónyi and K. Szlachányi, Phys. Lett. 98B (1981) 199.
[8] K. Kajantic, C. Montonen and E. Pietarinen, Z. Phys. C9 (1981) 253.

[9] M. Creutz, Phys. Rev. Lett. 45 (1980) 313.

[10] G. Bhanot and C. Rebbi, Nucl. Phys. B1 80 [FS2] (1981) 469.

[11] L. Susskind, Phys. Rev. D16 (1977) 3031.

[12] K. Wilson, Phys. Rev. D10 (1974) 2445; in: New phenomena in subnuclear physics (Erice, 1975) ed. A. Zichichi (Plenum, New York, 1977).

[13] S.D. Drell, M. Weinstein and S. Yankielowicz, Phys. Rev. D14 (1976) 487, 1627 ; Phys. Rev. D16 (1977) 1769.

[14] N.H. Christ, R. Friedberg and T.D. Lee, Random lattice ficld theory: general formulation, Columbia University preprint, CU-TP-205.

[15] C.B. Lang and H. Nicolai, Nucl. Phys. B200 [FS4] (1982) 135;

A. Hasenfratz and P. Hasenfratz, Phys. Lett. 104B (1981) 489 .

[16] T. Matthews and A. Salam, Nuovo Cimento 12 (1954) $563 ; 2$ (1955) 120.

[17] D. Weingarten, Phys. Lett. 109B (1982) 57.

[18] J. Engels, F. Karsch and H. Satz, Finite size effects in euclidean lattice thermodynamics for non-interacting Bose and Fermi systems, Bielefeld preprint, BI-TP $81 / 28$ (November 1981) to be published in Nucl. Phys. B [FS].

[19] A. Hasenfratz, P. Hasenfratz, Z. Kunszt and C.B. Lang, Phys. Lett. 110B (1982) 289.

[20] N. Kawamoto, Nucl. Phys. B190 [FS3] (1981) 617.

[21] F. Karsch, $\mathrm{SU}(N)$ gauge theory couplings on asymmetric lattices, Bielefeld preprint, BI-TP $81 / 34$ (November 1981), to be published in Nucl. Phys. B [FS].

[22] C.B. Lang and H. Nicolai, Nucl. Phys. B200 [FS4] (1982) 135.

[23] E.V. Shuryak, Phys. Lett. 107B (1981) 103; R.D. Pisarski, Phenomenology of the chiral phase transition, California University preprint, NSF-ITP-81-92 (1981). 